\title{
Near-Field Radiative Heat Transfer Eigenmodes
}

\author{
Stephen Sanders $\odot,{ }^{1}$ Lauren Zundel $\odot,{ }^{1}$ Wilton J. M. Kort-Kamp, ${ }^{2}$ \\ Diego A. R. Dalvit, ${ }^{2}$ and Alejandro Manjavacas $\oplus^{3,1, *}$ \\ ${ }^{1}$ Department of Physics and Astronomy, University of New Mexico, Albuquerque, New Mexico 87106, USA \\ ${ }^{2}$ Theoretical Division, Los Alamos National Laboratory, Los Alamos, New Mexico 87545, USA \\ ${ }^{3}$ Instituto de Óptica (IO-CSIC), Consejo Superior de Investigaciones Científicas, 28006 Madrid, Spain
}

(Received 11 February 2021; accepted 12 April 2021; published 12 May 2021)

\begin{abstract}
The near-field electromagnetic interaction between nanoscale objects produces enhanced radiative heat transfer that can greatly surpass the limits established by far-field blackbody radiation. Here, we present a theoretical framework to describe the temporal dynamics of the radiative heat transfer in ensembles of nanostructures, which is based on the use of an eigenmode expansion of the equations that govern this process. Using this formalism, we identify the fundamental principles that determine the thermalization of collections of nanostructures, revealing general but often unintuitive dynamics. Our results provide an elegant and precise approach to efficiently analyze the temporal dynamics of the near-field radiative heat transfer in systems containing a large number of nanoparticles.
\end{abstract}

DOI: 10.1103/PhysRevLett.126.193601

The thermal radiation exchanged between macroscopic bodies separated by macroscopic distances is accurately described by Planck's law [1]. However, this description breaks down when the distance between objects or their size becomes significantly smaller than the so-called thermal wavelength, which, for a temperature $T$, is $\lambda_{T}=2 \pi \hbar c /\left(k_{B} T\right)$. In this limit, the contribution of nearfield components of the electromagnetic field [2-10], together with the strong responses provided by the electromagnetic resonances of nanostructures [11-18], results in enhanced radiative heat transfer (RHT), which can surpass the blackbody limit by several orders of magnitude [19-23].

Near-field RHT is usually described within the framework of fluctuational electrodynamics $[23,24]$. In particular, when considering collections of nanostructures, a dipole approximation, where each nanoparticle is modeled as a fluctuating dipole, can be exploited [16,23,25-27]. By doing so, it is possible to calculate the power transferred between the different constituents for a particular fixed distribution of temperatures [16,28-30]. However, if one is interested in understanding the temporal evolution of the particle temperatures, this approach presents several disadvantages. Specifically, since the power transferred between the particles depends on their temperatures, which change over time, it is necessary to perform a new calculation at each step in the temporal evolution [31-34]. As a result, this approach provides little insight into the fundamental principles that determine the thermalization dynamics, requires separate calculations for each initial condition, and, in addition, can be computationally unfeasible when the number of particles is sufficiently large.

In this Letter, we present a different approach to describe the thermalization dynamics of ensembles of nanoparticles.
Our approach is based on linearizing the equations that govern the power transferred between the nanoparticles, which allows us to convert them into an eigenvalue problem. By doing so, we find a set of RHT eigenmodes for the ensemble, which completely describe the evolution of the system under any possible initial temperature distribution. Eigenmode expansions have been applied to a vast range of topics as a way to reveal physical insight [35-39]. Here, using this approach, we identify the general principles that control the thermalization process mediated by near-field RHT, which often give rise to unintuitive behaviors. This insight leads us to explore exotic scenarios, including dynamics in which the temperature of a particle oscillates around the equilibrium temperature as it thermalizes. The simplicity of this formalism makes it an elegant and efficient method to describe the dynamics of the nearfield RHT in ensembles with many nanoparticles.

We consider an ensemble of $N$ nanospheres with radii $R_{i}$ and temperatures $T_{i}$, placed at positions $\mathbf{r}_{i}$ and surrounded by vacuum at $T_{0}$, which we fix to $300 \mathrm{~K}$ for the remainder of this Letter. We assume that, for all particles, $R_{i} \ll \lambda_{T}$ and all interparticle distances $d_{i j}=\left|\mathbf{r}_{i}-\mathbf{r}_{j}\right| \geq 4 \max \left(R_{i}, R_{j}\right)$, but significantly smaller than $\lambda_{T}$. Therefore, we model the nanoparticles as fluctuating dipoles with electric polarizabilities $\alpha_{i}$. Following previous works [25-27,31], the power absorbed by particle $i$ is (see Ref. [40] for details)

$$
\mathcal{P}_{i}=\sum_{j=1}^{N} \int_{0}^{\infty} d \omega f_{i j}(\omega)\left[n\left(\omega, T_{j}\right)-n\left(\omega, T_{0}\right)\right],
$$

where $n(\omega, T)=\left[\exp \left(\hbar \omega / k_{B} T\right)-1\right]^{-1}$ is the Bose-Einstein distribution and $f_{i j}(\omega)=(2 \hbar \omega / \pi) \operatorname{Tr}\left[\operatorname{Im}\left\{\mathbf{A}_{i j} \operatorname{Im}\left\{\chi_{j}\right\} \mathbf{C}_{i j}^{+}\right\}\right]$. 
In this expression, "+" represents the conjugate transpose, the trace is taken over Cartesian components, and the different matrices, with dimensions $3 N \times 3 N$, are defined as $\mathbf{A}=[\mathcal{I}-\boldsymbol{\alpha} \mathbf{G}]^{-1}, \mathbf{C}=\left(\mathbf{G}+\mathbf{G}^{0}\right) \mathbf{A}$, and $\chi=\boldsymbol{\alpha}-\mathbf{G}^{0} \boldsymbol{\alpha}^{+} \boldsymbol{\alpha}$, with $\mathcal{I}$ being the identity matrix, $\boldsymbol{\alpha}$ a matrix with the polarizabilities, $\mathbf{G}$ the dipole-dipole interaction tensor, and $\mathbf{G}^{0}=\left(2 i \omega^{3} / 3 c^{3}\right) \mathcal{I}$. This model can be generalized to particles with magnetic response by including a magnetic polarizability $[16,30]$.

The temporal evolution of the temperatures of the nanoparticles is determined by the ratio between the power they absorb $\mathcal{P}_{i}$ and their heat capacities $\gamma_{i}$. By expanding $n\left(\omega, T_{j}\right)$ around $T_{0}$, as $n\left(\omega, T_{j}\right) \approx n\left(\omega, T_{0}\right)+\Delta T_{j} \partial n(\omega, T) /\left.\partial T\right|_{T=T_{0}}$, with $\Delta T_{j}=T_{j}-T_{0}$, we can linearize Eq. (1) to obtain the differential equation governing the evolution of the nanoparticle temperatures:

$$
\frac{d}{d t} \Delta \mathbf{T}(t)=-\mathbf{H} \Delta \mathbf{T}(t)
$$

Here, $\mathbf{H}=\boldsymbol{\Gamma}^{-1} \mathbf{F}$ is the product of the inverse of a diagonal matrix $\Gamma$ containing the heat capacities of the nanoparticles $\gamma_{i}$ and a symmetric matrix $\mathbf{F}$ with components

$$
F_{i j}=-\left.\int_{0}^{\infty} d \omega f_{i j}(\omega) \frac{\partial n(\omega, T)}{\partial T}\right|_{T=T_{0}} .
$$

As shown in Ref. [40], the structure of $\mathbf{H}$ ensures its diagonalizability. This allows us to write the solution of Eq. (2) using its eigenvalues $\lambda_{\mu}$ and eigenvectors $\Delta \mathcal{T}^{(\mu)}$ as

$$
\Delta \mathbf{T}(t)=\sum_{\mu=1}^{N} a_{\mu} e^{-\lambda_{\mu} t} \Delta \mathcal{T}^{(\mu)},
$$

where the coefficients $a_{\mu}$ are obtained from the weighted inner product between $\Delta \mathcal{T}^{(\mu)}$ and the vector containing the initial temperatures $\Delta \mathbf{T}(0)$ as $a_{\mu}=\sum_{i=1}^{N} \gamma_{i} \Delta T_{i}(0) \Delta \mathcal{T}_{i}^{(\mu)}$, with the eigenvectors satisfying $\sum_{i=1}^{N} \gamma_{i} \Delta \mathcal{T}_{i}^{(\mu)} \Delta \mathcal{T}_{i}^{(\nu)}=\delta_{\mu \nu}$. Therefore, we conclude from Eq. (3) that the dynamics of the near-field RHT of an ensemble of nanoparticles can be completely understood by analyzing its RHT eigenmodes and decay rates given, respectively, by the eigenvectors and eigenvalues of $\mathbf{H}$. Importantly, $\mathbf{H}$ is positive definite (i.e., $\lambda_{\mu}>0$ ), which ensures that the ensemble thermalizes as $t \rightarrow \infty$.

This approach assumes that the temperature dependence of the material properties of the nanoparticles can be neglected. Furthermore, as discussed in Ref. [40], its accuracy improves as $\max \left(\left|\Delta T_{j}\right| / T_{0}\right)$ and $\hbar \omega_{0} /\left(k_{B} T_{0}\right)$ decrease. Here, $\omega_{0}$ represents the characteristic frequency of the electromagnetic response of the nanoparticles. For the systems under consideration, the results of the eigenmode approach have very good agreement with the
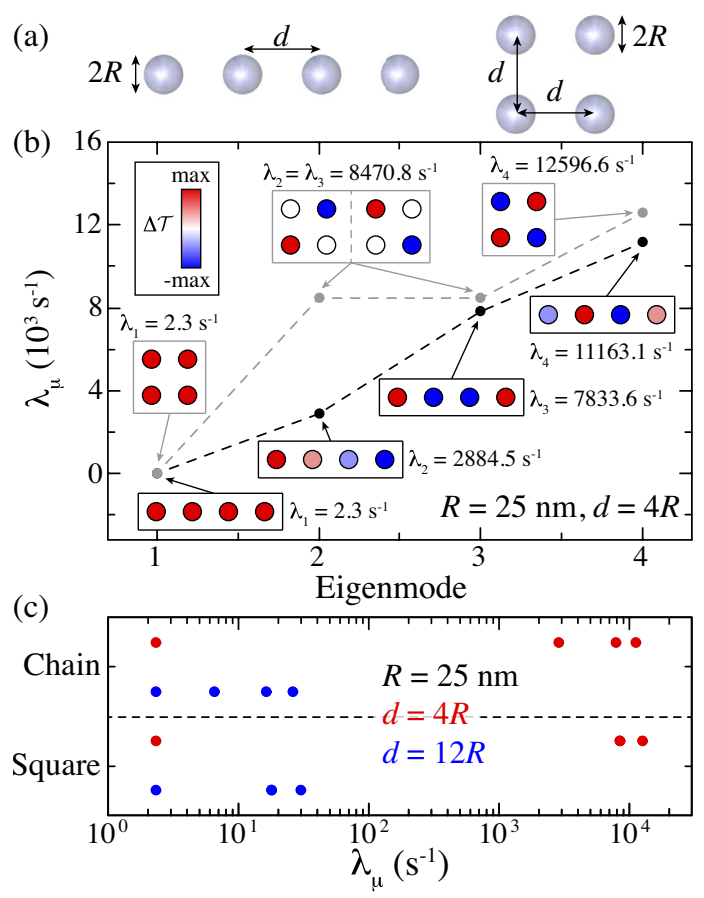

FIG. 1. (a) Schematics of the systems under study. (b) Decay rates of the RHT eigenmodes of the two systems assuming $R=25 \mathrm{~nm}$ and $d=4 R$. The insets display the components of the RHT eigenmodes and the value of the associated decay rate. (c) Decay rates for different values of $d$.

nonlinearized full calculation up to $\max \left(\left|\Delta T_{j}\right| / T_{0}\right) \approx 1 / 3$, as shown in Ref. [40].

To illustrate the developed framework, we consider a simple example, although the conclusions we draw are general to any ensemble of nanoparticles. In particular, we analyze the two systems depicted in Fig. 1(a), consisting of $N=4$ identical $\mathrm{SiC}$ spherical nanoparticles arranged in either a chain or a square (see Ref. [40] for a similar analysis of a system with $N=2197$ ). We obtain the polarizability of the particles from the dipolar Mie coefficient [44] using the dielectric function $\varepsilon(\omega)=$ $\varepsilon_{\infty}\left[1+\left(\omega_{L}^{2}-\omega_{T}^{2}\right) /\left(\omega_{T}^{2}-\omega^{2}-i \omega \tau^{-1}\right)\right]$, with $\varepsilon_{\infty}=6.7$, $\hbar \omega_{T}=98.3 \mathrm{meV}, \hbar \omega_{L}=120 \mathrm{meV}$, and $\hbar \tau^{-1}=0.59 \mathrm{meV}$ [45]. Figure 1(b) analyzes the RHT eigenmodes of the chain (black) and the square (gray) assuming that the particles have a radius $R=25 \mathrm{~nm}$ and are separated by $d=4 R$. The chain has four distinct eigenmodes, while the larger symmetry of the square results in two of its modes being degenerate. Since particles with the same temperature do not exchange heat with one another, every ensemble, including the two analyzed here, must always have an eigenmode with equal amplitude in all particles. This eigenmode, which we label as $\mu=1$, represents a net transfer of heat between the ensemble and the environment and, as explained below, always has the slowest decay rate. The orthogonality of the eigenmodes forces the rest of them to satisfy $\sum_{i=1}^{N} \gamma_{i} \Delta \mathcal{T}_{i}^{(\mu>1)}=0$, which physically means 
that they represent processes in which the heat stored in the ensemble remains constant. Therefore, every eigenmode with $\mu>1$ describes a near-field RHT process among the nanoparticles of the ensemble. Examining the components of these eigenmodes, we observe that, as $\mu$ increases, the length scale over which the sign of the components alternates, and hence the near-field RHT occurs, decreases. This is consistent with the increase of the associated decay rate, whose value is dominated by terms proportional to $\left(R_{i} R_{j}\right)^{3} / d_{i j}^{6}$. In contrast, $\lambda_{1}$ describes the net radiation exchange between the ensemble and the environment, which scales as $\left(R_{i} / \lambda_{T}\right)^{3}$. Therefore, for nearfield RHT (i.e., $d_{i j} \ll \lambda_{T}$ ), $\lambda_{1}$ always has the smallest value among all of the decay rates, although, as shown in Fig. 1(c), the difference between $\lambda_{1}$ and the rest of the decay rates is reduced by increasing the distance between the particles.

We know from Eq. (3) that the thermalization of an ensemble of particles is initially dominated by the eigenmodes with largest decay rates. However, for sufficiently long time, this process is controlled by the first eigenmode, which, as discussed above, has equal amplitude in all particles and, consequently, its decay rate is the smallest. Therefore, in the limit $t \rightarrow \infty$, the thermalization dynamics of a given ensemble depends exclusively on $a_{1} \propto \sum_{i=1}^{N} \gamma_{i} \Delta T_{i}(0)$, or, in other words, the total heat initially stored in it. This gives rise to interesting behaviors, as illustrated in Fig. 2(a). There, we analyze the thermalization dynamics of a square array of $N=49$ identical $\mathrm{SiC}$ particles with $R=25 \mathrm{~nm}$ and $d=4 R$ (solid curves). We consider different initial temperature distributions, all of them corresponding to the same value of $a_{1}$. Specifically, the gray curve displays the evolution of the temperature of the nanoparticles when all of them begin at $\Delta T=1 \mathrm{~K}$. On the other hand, the colored curves represent different scenarios where only one particle, indicated in the schematics using the same color, is initially hot at $\Delta T=49 \mathrm{~K}$. One might anticipate that when all of the particles begin at $\Delta T=1 \mathrm{~K}$, the system would thermalize most quickly to the environment. However, as seen in Fig. 2(a), this is not the case. Instead, in all of the scenarios under consideration, all of the particles approach the equilibrium identically as $e^{-\lambda_{1} t}$.

Interestingly, for the scenarios in which only one particle is initially hot, the thermalization process happens over two steps: first, all of the particles converge to $\Delta T=1 \mathrm{~K}$, and, second, the whole array thermalizes to the environment. This behavior is the result of the large difference between $\lambda_{1}$ and the rest of the decay rates, as shown in Ref. [40]. Therefore, if such difference is decreased by, for instance, increasing the interparticle distance to $d=12 R$, the twostep behavior fades away, as shown by the dashed curves.

Although, so far, we have only considered ordered distributions of particles, our conclusions apply to any arbitrary ensemble of particles. For example, in Fig. 2(b), we consider an ensemble of $N=490$ identical $\mathrm{SiC}$

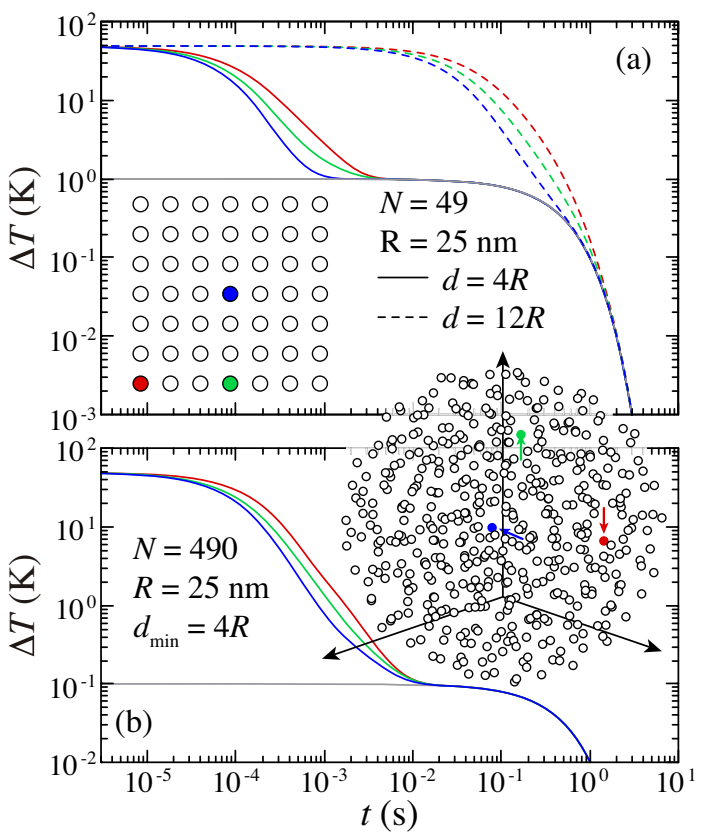

FIG. 2. (a) Thermalization dynamics for an array of $N=49$ SiC nanoparticles with $R=25 \mathrm{~nm}$ under different initial conditions. The colored curves display the evolution of the temperature of the particle of that color in the inset schematics, when such particle is initially at $\Delta T=49 \mathrm{~K}$ and the rest at $\Delta T=0 \mathrm{~K}$. The gray curves represent the case where all of the particles are initially at $\Delta T=49 / N \mathrm{~K}$. In all cases, solid and dashed curves correspond to $d=4 R$ and $d=12 R$. (b) Same as (a), but for an ensemble of $N=490 \mathrm{SiC}$ nanoparticles with $R=25 \mathrm{~nm}$, randomly distributed inside a spherical volume of radius $600 \mathrm{~nm}$ with a minimum interparticle distance $d_{\min }=4 R$ (see schematics).

nanoparticles with $R=25 \mathrm{~nm}$ randomly arranged within a spherical volume of radius $600 \mathrm{~nm}$, as shown in the inset. As in Fig. 2(a), we compare the thermalization process for four different initial conditions; in three of them, one particle, marked in the schematics with the same color as its corresponding curve, begins at $\Delta T=49 \mathrm{~K}$, while, in the fourth (gray curve), all of the particles begin at $\Delta T=0.1 \mathrm{~K}$. As expected, since $a_{1}$ takes the same value for all of the cases, they all approach the thermalization to the environment identically, despite their very different initial temperature distributions.

Another interesting scenario to consider is when the initial distribution of temperatures is orthogonal to the first RHT eigenmode and, hence, $a_{1}=0$. Physically, this means that, although the system is not thermalized, the total amount of heat initially stored in it is zero. In this case, the thermalization process is governed entirely by the eigenmodes describing the near-field RHT between the particles, since a net transfer of heat to the environment (described by the first RHT eigenmode) is forbidden. To illustrate this, in Fig. 3(a), we study the thermalization dynamics of the array of Fig. 2(a) with $d=4 R$, for the initial temperature distributions depicted in the insets of 


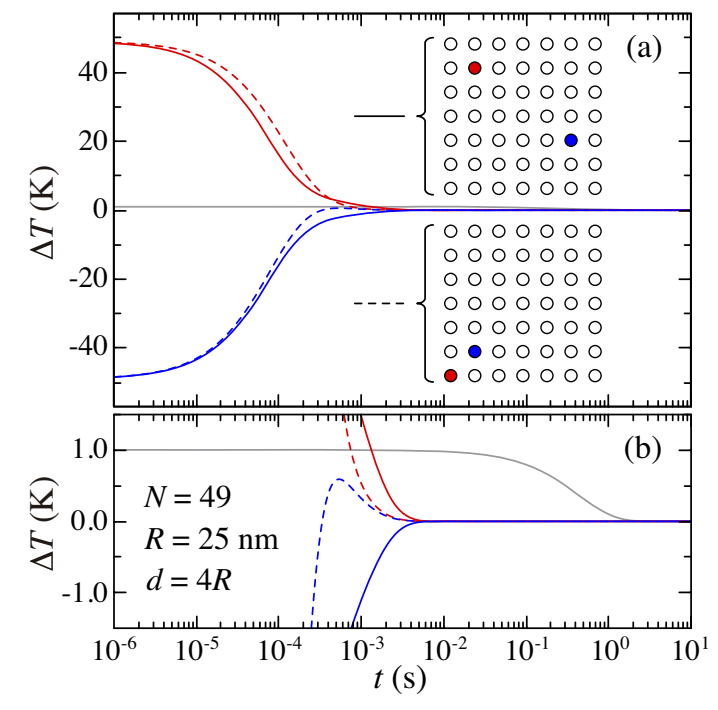

FIG. 3. (a) Thermalization dynamics for a hot (red curves) and a cold (blue curves) particle in an array of $N=49 \mathrm{SiC}$ nanoparticles with $R=25 \mathrm{~nm}$ and $d=4 R$. The red and blue particles are initially at $\Delta T=49 \mathrm{~K}$ and $\Delta T=-49 \mathrm{~K}$, respectively, while the rest are at $\Delta T=0 \mathrm{~K}$. We consider the two cases depicted in the schematics, which are displayed with solid and dashed curves, respectively. For comparison, the gray curve represents the thermalization dynamics when all of the particles are initially at $\Delta T=49 / N \mathrm{~K}$. (b) Enlargement of (a) around $\Delta T=0 \mathrm{~K}$.

Fig. 3(a). In both of them, one particle begins at $\Delta T=$ $49 \mathrm{~K}$ and another at $\Delta T=-49 \mathrm{~K}$, while the rest of the array is at $\Delta T=0 \mathrm{~K}$, so $a_{1}=0$. The corresponding results are displayed using solid and dashed curves, as indicated by the legend, with red and blue colors describing, respectively, the temperature of the hot and cold particles. As expected, in both cases, the thermalization of the array occurs on a timescale $\sim \lambda_{2}^{-1} \approx 10^{-3}$ s. This is much faster than the thermalization when all of the nanoparticles begin at $\Delta T=1 \mathrm{~K}$ (gray curve), even though, in that case, the particles have to undergo a temperature change of only $1 \mathrm{~K}$ [see Fig. 3(b) for an enlargement around $\Delta T=0 \mathrm{~K}$ ]. The reason is, again, the large difference between $\lambda_{\mu>1}$ and $\lambda_{1}$.

Interestingly, the closer look provided in Fig. 3(b) reveals an unituitive behavior: when the hot and cold particles are next to each other (dashed curves), the temperature of the initially cold particle rises beyond $\Delta T=$ $0 \mathrm{~K}$ and subsequently approaches it from above. We attribute this behavior to the difference in the local environment of the two nanoparticles; while the hot one lies on the corner of the array, the cold one is situated in the interior and is therefore surrounded by more particles. This creates an imbalance in the cooling and heating rates of the two particles.

We can use the RHT eigenmode framework to gain more insight into this oscillatory behavior. To that end, we analyze a simpler system that exhibits similar oscillatory dynamics but in a more pronounced way. In particular, we consider the chain of $N=5 \mathrm{SiC}$ nanoparticles with

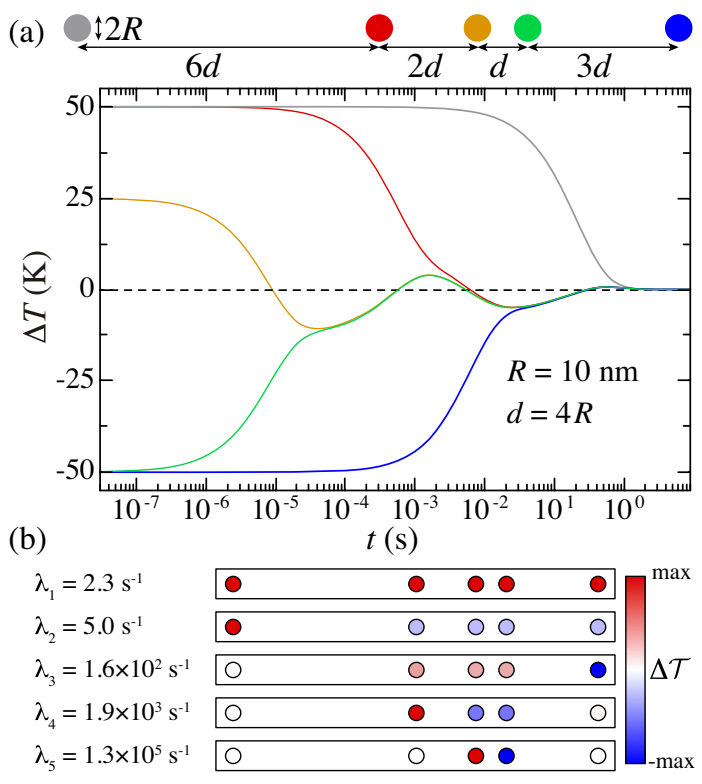

FIG. 4. (a) Thermalization dynamics for a chain with $N=5$ $\mathrm{SiC}$ nanoparticles arranged as shown in the schematics. We assume that $R=10 \mathrm{~nm}, d=4 R$, and the nanoparticles are initially at $\Delta T=50,50,25,-50$, and $-50 \mathrm{~K}$. (b) RHT eigenmodes of the chain and their associated decay rates.

$R=10 \mathrm{~nm}$ and $d=4 R$, shown in the schematics of Fig. 4. The particles are initially at $\Delta T=50,50,25,-50$, and $-50 \mathrm{~K}$. The different curves in Fig. 4(a) show the evolution of the temperature of the particle with matching color. As the particles thermalize, their temperatures oscillate around $\Delta T=0 \mathrm{~K}$, with the center one (yellow) crossing this value 4 times throughout the process. The origin of this exotic behavior becomes clear by considering the RHT eigenmodes of the system, which are shown, with their corresponding decay rates, in Fig. 4(b). Specifically, the initial stage of the thermalization is dominated by the eigenmode with the largest decay rate, which corresponds to a nearfield RHT process happening almost exclusively between the center nanoparticle and its nearest neighbor. After that, the contribution of the next fastest eigenmode drives the thermalization of both of those particles with their nextnearest neighbor. This pattern repeats with each successive eigenmode, resulting in the observed oscillatory behavior of $\Delta T$.

In conclusion, we have presented a theoretical framework to characterize the temporal dynamics of the nearfield RHT in arbitrary ensembles of nanoparticles. Our approach is based on an eigenmode expansion of the equations that govern the RHT, obtained upon their linearization. The resulting set of eigenmodes completely characterize the RHT between the constituents of the ensemble and their environment and therefore allow us to express, in a closed form, the evolution of the temperatures of the particles for any initial condition. Exploiting this formalism, we have identified general characteristics of the dynamics of RHT, which often present themselves in 
unintuitive ways. Specifically, we have shown that an ensemble of nanoparticles beginning with a fixed amount of stored heat always approaches thermalization identically, regardless of how that heat is initially distributed. Similarly, when the total initial heat stored in an ensemble is zero, the system reaches thermal equilibrium faster than the case where there is any initially stored heat. We have also predicted and explained an exotic behavior in which the temperature of nanoparticles oscillates around the equilibrium value as they thermalize. Our results provide an insightful and computationally efficient approach to study the thermalization dynamics mediated by the near-field RHT, which will facilitate the systematic investigation of the impact that novel phenomena, such as topology [46] and nonreciprocity $[47,48]$, have on this process. Furthermore, this framework can be exploited to analyze the combined transfer of energy and momentum mediated by the fluctuations of the electromagnetic field [39].

This work has been sponsored by the U.S. National Science Foundation (Grant No. DMR-1941680) and the Ministerio de Ciencia, Innovacin y Universidades of Spain (Grant No. TEM-FLU PID2019-109502GA-I00). L.Z. acknowledges support from the Department of Energy Computational Science Graduate Fellowship (Grant No. DE-SC0020347). D. A. R. D. and W. J. M. K.-K. acknowledge financial support from the Laboratory Directed Research and Development program of Los Alamos National Laboratory under LDRD Project No. 20210327ER.

* Corresponding author. a.manjavacas@csic.es

[1] F. Reif, Fundamentals of Statistical and Thermal Physics (McGraw-Hill, New York, 1965).

[2] A. Narayanaswamy, S. Shen, L. Hu, X. Chen, and G. Chen, Appl. Phys. A 96, 357 (2009).

[3] E. Rousseau, A. Siria, G. Jourdan, S. Volz, F. Comin, J. Chevrier, and J. J. Greffet, Nat. Photonics 3, 514 (2009).

[4] R. S. Ottens, V. Quetschke, S. Wise, A. A. Alemi, R. Lundock, G. Mueller, D. H. Reitze, D. B. Tanner, and B. F. Whiting, Phys. Rev. Lett. 107, 014301 (2011).

[5] R. St-Gelais, B. Guha, L. Zhu, S. Fan, and M. Lipson, Nano Lett. 14, 6971 (2014).

[6] H. Chalabi, E. Hasman, and M. L. Brongersma, Phys. Rev. B 91, 014302 (2015).

[7] K. Kim, B. Song, V. Fernández-Hurtado, W. Lee, W. Jeong, L. Cui, D. Thompson, J. Feist, M. T. H. Reid, F. J. GarcíaVidal et al., Nature (London) 528, 387 (2015).

[8] B. Song, D. Thompson, A. Fiorino, Y. Ganjeh, P. Reddy, and E. Meyhofer, Nat. Nanotechnol. 11, 509 (2016).

[9] R. St-Gelais, L. Zhu, S. Fan, and M. Lipson, Nat. Nanotechnol. 11, 515 (2016).

[10] K. Shi, Y. Sun, Z. Chen, N. He, F. Bao, J. Evans, and S. He, Nano Lett. 19, 8082 (2019).

[11] G. Domingues, S. Volz, K. Joulain, and J. J. Greffet, Phys. Rev. Lett. 94, 085901 (2005).
[12] A. I. Volokitin and B. N. J. Persson, Rev. Mod. Phys. 79, 1291 (2007).

[13] P. Ben-Abdallah, K. Joulain, J. Drevillon, and C. Le Goff, Phys. Rev. B 77, 075417 (2008).

[14] A. Narayanaswamy and G. Chen, Phys. Rev. B 77, 075125 (2008).

[15] G. V. Dedkov and A. A. Kyasov, J. Comput. Theor. Nanosci. 7, 2019 (2010).

[16] A. Manjavacas and F. J. García de Abajo, Phys. Rev. B 86, 075466 (2012).

[17] A. Manjavacas, S. Thongrattanasiri, J. J. Greffet, and F. J. García de Abajo, Appl. Phys. Lett. 105, 211102 (2014).

[18] F. V. Ramirez, S. Shen, and A. J. H. McGaughey, Phys. Rev. B 96, 165427 (2017).

[19] M. P. Bernardi, D. Milovich, and M. Francoeur, Nat. Commun. 7, 12900 (2016).

[20] R. Yu, A. Manjavacas, and F. J. García de Abajo, Nat. Commun. 8, 2 (2017).

[21] A. Fiorino, D. Thompson, L. Zhu, R. Mittapally, S.-A. Biehs, O. Bezencenet, N. El-Bondry, S. Bansropun, P. Ben-Abdallah, E. Meyhofer et al., ACS Nano 12, 5774 (2018).

[22] J. C. Cuevas and F. J. Garca-Vidal, ACS Photonics 5, 3896 (2018)

[23] S.-A. Biehs, R. Messina, P. S. Venkataram, A. W. Rodriguez, J. C. Cuevas, and P. Ben-Abdallah, arXiv:2007.05604v1.

[24] D. Polder and M. Van Hove, Phys. Rev. B 4, 3303 (1971).

[25] P. Ben-Abdallah, S. A. Biehs, and K. Joulain, Phys. Rev. Lett. 107, 114301 (2011).

[26] M. Nikbakht, J. Appl. Phys. 116, 094307 (2014).

[27] M. Nikbakht, Europhys. Lett. 110, 14004 (2015).

[28] P. Ben-Abdallah, A. Belarouci, L. Frechette, and S.-A. Biehs, Appl. Phys. Lett. 107, 053109 (2015).

[29] J. Dong, J. Zhao, and L. Liu, J. Quant. Spectrosc. Radiat. Transfer 197, 114 (2017).

[30] J. Dong, J. Zhao, and L. Liu, Phys. Rev. B 95, 125411 (2017)

[31] R. Messina, M. Tschikin, S.-A. Biehs, and P. Ben-Abdallah, Phys. Rev. B 88, 104307 (2013).

[32] Y. Wang and J. Wu, AIP Adv. 6, 025104 (2016).

[33] J. Song, L. Lu, B. Li, B. Zhang, R. Hu, X. Zhou, and Q. Cheng, Int. J. Heat Mass Transfer 150, 119346 (2020).

[34] L. Zundel and A. Manjavacas, Phys. Rev. Applied 13, 054054 (2020).

[35] M. I. Hussein, Proc. R. Soc. A 465, 2825 (2009).

[36] R. Yu, L. M. Liz-Marzán, and F. J. García de Abajo, Chem. Soc. Rev. 46, 6710 (2017).

[37] Z. Lu and O. Raz, Proc. Natl. Acad. Sci. U.S.A. 114, 5083 (2017).

[38] V. V. Kocharovsky, C. B. Reynolds, and V. V. Kocharovsky, Phys. Rev. A 100, 053854 (2019).

[39] S. Sanders, W. J. M. Kort-Kamp, D. A. R. Dalvit, and A. Manjavacas, Commun. Phys. 2, 71 (2019).

[40] See Supplemental Material at http://link.aps.org/ supplemental/10.1103/PhysRevLett.126.193601, which includes Refs. [41-43], for the derivation of Eq. (1), an analysis of the diagonalizability of the matrix $\mathbf{H}$, and three supplemental figures. 
[41] S. M. Rytov, Theory of Electric Fluctuations and Thermal Radiation (Air Force Cambridge Research Center, Bedford, MA, 1959).

[42] A. Manjavacas and F. J. García de Abajo, Phys. Rev. Lett. 105, 113601 (2010).

[43] S. Friedberg, A. Insel, and L. Spence, Linear Algebra, Featured Titles for Linear Algebra (Advanced) Series (Pearson Education, Upper Saddle River, 2003).

[44] V. Myroshnychenko, J. Rodríguez-Fernández, I. PastorizaSantos, A. M. Funston, C. Novo, P. Mulvaney, L. M.
Liz-Marzán, and F. J. García de Abajo, Chem. Soc. Rev. 37, 1792 (2008).

[45] E. D. Palik, Handbook of Optical Constants of Solids (Academic Press, San Diego, 1985).

[46] A. Ott and S.-A. Biehs, Phys. Rev. B 102, 115417 (2020).

[47] L. Zhu and S. Fan, Phys. Rev. Lett. 117, 134303 (2016).

[48] A. Ott, R. Messina, P. Ben-Abdallah, and S.-A. Biehs, Appl. Phys. Lett. 114, 163105 (2019). 